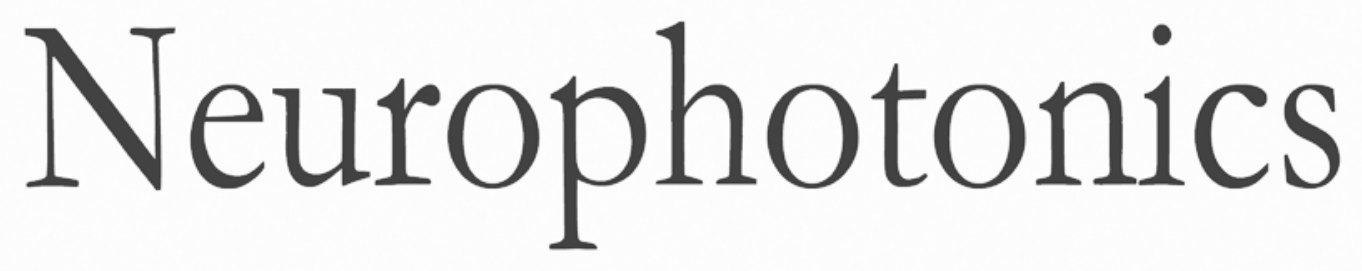

\title{
From brain to blood vessels and back: a noninvasive optical imaging approach
}

Gabriele Gratton

Antonio M. Chiarelli

Monica Fabiani 


\title{
From brain to blood vessels and back: a noninvasive optical imaging approach
}

\author{
Gabriele Gratton,, ${ }^{a, b, \star}$ Antonio M. Chiarelli, ${ }^{b}$ and Monica Fabiani $i^{a, b}$ \\ aUniversity of Illinois at Urbana Champaign, Psychology Department, Champaign, Illinois, United States \\ bUniversity of Illinois at Urbana Champaign, Beckman Institute, Urbana, Illinois, United States
}

\begin{abstract}
The seminal work of Grinvald et al. has paved the way for the use of intrinsic optical signals measured with reflection methods for the analysis of brain function. Although this work has focused on the absorption signal associated with deoxygenation, due to its detailed mapping ability and good signal-to-noise ratio, Grinvald's group has also described other intrinsic signals related to increased blood flow, scattering effects directly related to neural activation, and pulsation effects related to arterial function. These intrinsic optical signals can also be measured using noninvasive diffuse optical topographic and tomographic imaging (DOT) methods that can be applied to humans. Here we compare the reflection and DOT methods and the evidence for each type of intrinsic signal in these two domains, with particular attention to work that has been conducted in our laboratory. This work reveals the refined two-way relationship that exists between vascular and neural phenomena in the brain: arterial health is related to normal brain structure and function, both across individuals and across brain regions within an individual, and neural function influences blood flow to specific cortical regions. DOT methods can provide quantitative tools for investigating these relationships in normal human subjects. $\odot 2017$ Society of PhotoOptical Instrumentation Engineers (SPIE) [DOI: 10.1117/1.NPh.4.3.031208]
\end{abstract}

Keywords: reflection optical imaging; diffuse optical tomography; intrinsic optical signal; deoxygenation signal; oxygenation signal; fast optical signals; optical measures of cerebrovascular pulse (pulse-DOT); neurovascular coupling.

Paper 17007SSVR received Jan. 15, 2017; accepted for publication Mar. 10, 2017; published online Apr. 7, 2017.

\section{Introduction: Imaging Light Reflection from the Exposed Cortex}

In this paper, we review some of the work conducted in our laboratory on diffuse optical topographic and tomographic (DOT) imaging methods, and in particular work related to the development of noninvasive methodologies capitalizing on "intrinsic" optical signals (i.e., signals that can be observed without the introduction of external contrast agents) to study the bidirectional relationships between vascular and brain function. This work critically builds on the seminal research of Grinvald et al., ${ }^{1}$ who provided the first imaging method for studying changes in the optical properties of the brain associated with neural activity. The work presented in their 1986 paper was based on the measurement of a signal associated with changes in the oxygenation level of the blood perfusing active cortical regions. In their work, Grinvald et al. used a preparation in which a porthole was inserted in the skull of mammals (rats, cats, and monkeys) to expose the occipital cortex (although the dura was preserved to minimize movement of the brain surface due to respiration and arterial pulsation). Using widespread illumination and a CCD recording camera, they measured changes in the reflection of light from the surface of the cortex with a temporal resolution (TR) of a few hundred $\mathrm{ms}$ and a spatial resolution of $\sim 50 \mu \mathrm{m} .^{2,3}$ This and subsequent work [Refs. 2-4; see also Refs. 5-7] demonstrated that in fact a number of different physiological signals, some of which are related to neural

${ }^{\star}$ Address all correspondence to: Gabriele Gratton, E-mail: grattong@illinois .edu function, are visible with this methodology. One of these signals is the pulsation of blood in the arteries, which produces a characteristic oscillatory activity, whose frequency is determined by the heart rate. In the original work, in which the focus is to reconstruct the functional architecture of the cortex, this phenomenon is considered as noise and needs to be eliminated in order to unmask the brain's response. Most importantly, using spectroscopic methods, three types of phenomena associated with neural activity were observed in active brain regions. ${ }^{2-4}$ The most rapid of these phenomena is associated with a change in the scattering of light from tissue (i.e., a broad spectral signal, slightly decreasing with wavelength) that closely tracks the period of visual stimulation. This signal is followed by a deoxygenation signal (with a spectral characteristic consistent with an increase in the concentration of deoxy-hemoglobin and a decrease in the concentration of oxy-hemoglobin), which is already visible $500 \mathrm{~ms}$ poststimulus, ${ }^{6,7}$ and reaches its peak within 3 to $4 \mathrm{~s}$ from the beginning of the visual stimulation. Finally, a larger signal appears with an onset latency of $2 \mathrm{~s}$ and a peak latency of $\sim 6$ to $7 \mathrm{~s}$, which indicates an increase in total hemoglobin concentration (reflecting an increase in blood flow), followed by a shift toward a higher oxygenation level. Whereas the first two of these neural-related signals are clearly localized to the parenchyma, the third of these signals originally appeared to extend to the veins. ${ }^{2-4}$

Subsequent research, however, found conflicting evidence regarding the possibility of reliably observing the early deoxygenation signal (also known as the "initial dip"). ${ }^{5-7}$ In addition, further research has indicated that the later signal, related to an increase in blood flow to activated areas, with 
a subsequent shift to greater blood oxygen saturation, may in fact possess both a high degree of spatial resolution and a relatively high signal-to-noise ratio (SNR), making it the most useful intrinsic signal for functional mapping of the brain. ${ }^{8-11}$ When measured with functional magnetic resonance imaging (fMRI), this signal is known as the blood oxygen leveldependent (BOLD) signal.

High-resolution spatial mapping based on intrinsic optical signals has provided tremendous insights into the functional organization of different cortical regions. For instance, current understanding of the functional organization of the primary visual cortex in primates, such as the existence of color pinwheels, etc., ${ }^{5}$ largely depends on information obtained through intrinsic optical imaging.

\section{Imaging Light Diffusion in the Intact Human Head}

In parallel with this animal research and partly inspired by the work of Grinvald et al., interest arose in the possibility of conducting noninvasive imaging studies in humans. A large part of this research is now conducted using fMRI methods utilizing the BOLD signal commonly observed with $\mathrm{T} 2 *$-weighted MRI, which, as mentioned, is largely consistent with the late increase in blood flow in active cortical regions reported by Grinvald's group. The optical counterpart of the BOLD signal in humans relies on diffuse (rather than reflection) optical methods, which quantify light injected into and detected from the scalp.

Diffuse optical imaging is an approach first proposed in the late 1970s by Jobsis. ${ }^{12}$ This technique is based on the idea that the main obstacle to image deep structures (such as the brain) in highly scattering media (such as the human head) is the strong reflection signal provided by the initial scattering episodes occurring in the most superficial layers of the media (typically in the first $5 \mathrm{~mm}$, including the epidermis, derma, and superficial bone layers). This signal is several orders of magnitude larger than the light signal from deeper structures, which are therefore rendered invisible. To reveal this signal, it is necessary to ignore the photons that are backscattered to the surface because of these initial scattering episodes. This is achieved by small points of illumination, and by measuring the light exiting from the medium at some distance from the source, after multiple scattering episodes may have occurred deep in the tissue, as characterized using the mathematics of diffusion processes (hence the term diffuse optical imaging). ${ }^{13,14}$ These measures are sensitive to optical phenomena occurring within volumes that have approximately the shape of curved spindles, whose size and position in the head are determined by the scalp location of the relevant source and detector pair (optode) on the scalp, as well as by the optical properties (scattering and absorption coefficients) of the head tissues. Using multiple sources and detectors, it is possible to reconstruct two-dimensional (diffuse optical topography, e.g., Ref. 15) or three-dimensional (3-D) maps (DOT, e.g., Ref. 16) of where the relevant optical phenomena occur within the brain.

Figure 1 reports a schematic comparing the principles of reflective and diffuse optical imaging. Compared to reflective imaging, diffuse optical imaging has both advantages and disadvantages. The main advantage is the much greater penetrationincreasing from a few $\mathrm{mm}$ to several $\mathrm{cm}$, making it possible to measure noninvasively optical phenomena occurring in the human brain. Another advantage is that, using an extended number of sources and detectors, the majority of the cortical mantle can be imaged at the same time. The main disadvantage is the substantial loss of spatial resolution, which is determined by the multiple scattering episodes the photons go through before reaching the detectors. Because of this, the spatial resolution drops from 50 to $100 \mu \mathrm{m}$ in reflective imaging ${ }^{1-3}$ to about 5 to $20 \mathrm{~mm}$ in diffuse optical imaging, ${ }^{14,18}$ with differences due to the exact methodology employed.

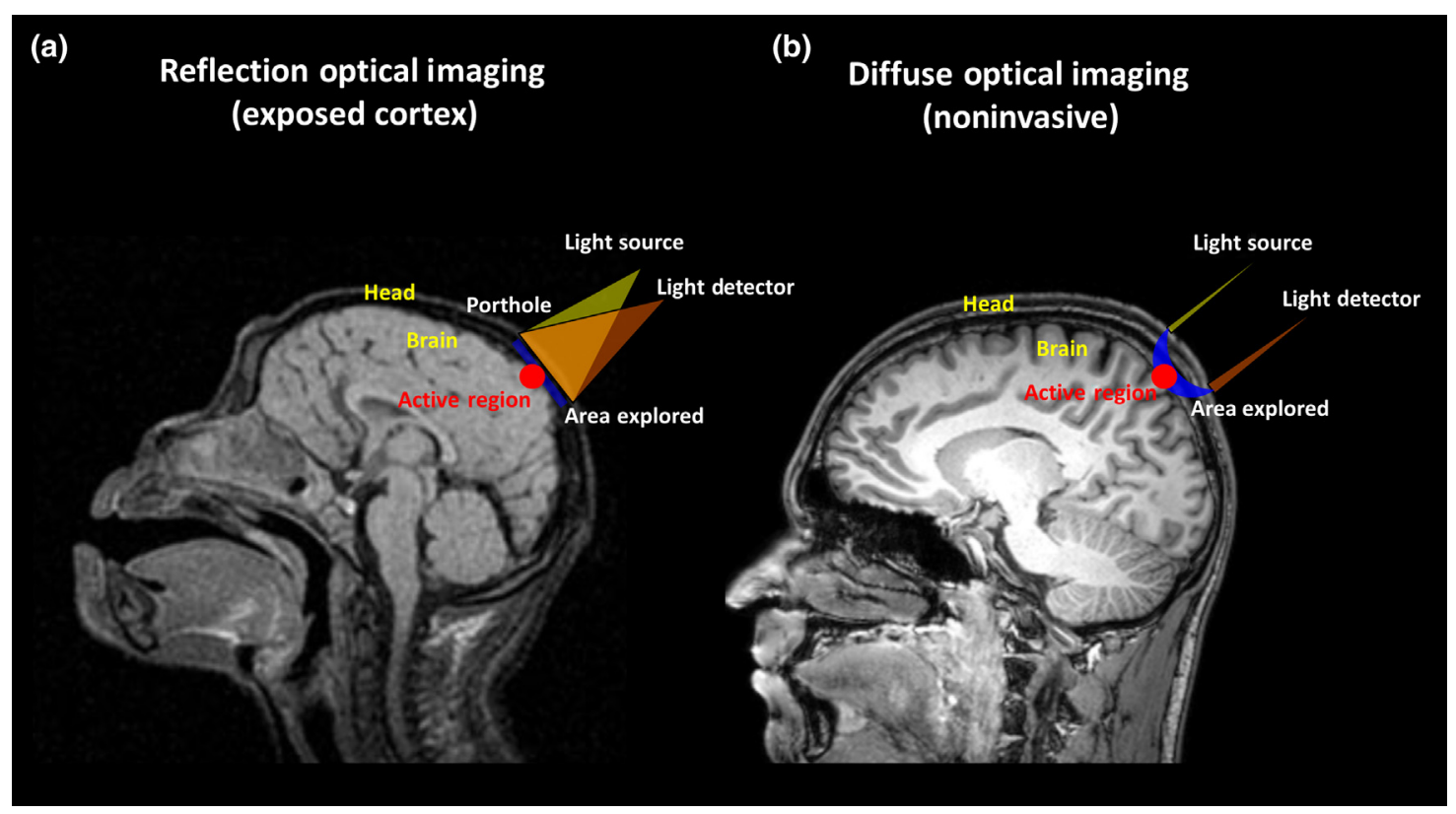

Fig. 1 Schematic representation comparing (a) exposed-cortex reflection optical imaging methods with (b) noninvasive diffuse optical imaging methods. The MR image of the monkey head presented in (a) is obtained from Ref. 17. 


\section{Signals Measurable with Diffuse Optical Imaging}

\subsection{Oxygenation signal}

Diffuse optical imaging methods have been employed for over 20 years. The first observation of a functional signal (an increase in the absorption of light in the visual cortex attributable to an increase in the concentration of oxy-hemoglobin and total hemoglobin during a mental task associated with the difficulty of the task) measurable in the human brain was obtained in 1993. ${ }^{19}$ This phenomenon appears to be closely related to the large oxygenation signal observed by Malonek and Grinvald ${ }^{3,4}$ and the BOLD response observed with fMRI. ${ }^{20}$

The oxygenation signal is relatively easy to observe and has been used extensively during the last 20 years as the main signal in functional near-infrared spectroscopy (fNIRS; for a review, see Ref. 21). This technology is commonly used to generate images of brain activity with a spatial resolution of $\sim 2 \mathrm{~cm}$, and a TR of several seconds. fNIRS is a less expensive, more portable, and more easily scalable technology than fMRI and can be applied to practically all populations. For these reasons, it is particularly useful for studying clinical and special populations that can be difficult to study with MRI, and/or in conditions in which the cost of recording may be an issue (e.g., when very extended recordings are required) or when a portable device is needed. fNIRS also allows for the relative and absolute quantification of the two hemoglobin species, which is currently not possible with fMRI. The main drawbacks of fNIRS are its limited penetration (about $30 \mathrm{~mm},{ }^{14}$ common to all diffuse optical imaging methods), its limited spatial resolution relative to fMRI and reflective optical imaging ( $2 \mathrm{~cm}$ when based on the amount of light emitted by a source and reaching a detector), and, in some cases, its sensitivity to movement artifacts.

\subsection{Deoxygenation signal}

To this date, demonstration with diffuse optical methods in humans of a relatively rapid (500 ms latency) deoxygenation signal (such as the one that is most commonly used for intrinsic exposed-cortex optical imaging studies, e.g., Refs. 6 and 7) has proven difficult. Some studies have indicated that the relatively slow oxygenation signal described in the previous paragraph may show different phases, but none of them appears to have onset latency shorter than $2 \mathrm{~s}$ (e.g., Ref. 22). In contrast, research in animals (such as song birds) ${ }^{23}$ has indicated the presence of deoxygenation signals with relatively short latency and clearly preceding the oxygenation response. It is not entirely clear why animal recordings are more prone than human recordings to show relatively rapid deoxygenation responses. One possibility is that the deoxygenation response, being very localized, may be less evident at the slow spatial resolution offered by fNIRS. However, it should be noted that fMRI studies also have difficulties revealing a rapid deoxygenation response, which should appear as a "dip" in the BOLD response (but see the research from Ugurbil's group in Minnesota ${ }^{24}$ ); however, most fMRI studies have too sparse a TR to be able to follow rapid changes as those associated with the deoxygenation response.

\subsection{Scattering signal}

Our lab has contributed to this area by showing that another functional signal, more directly related to neural activity, can be obtained in active cortex. Specifically, in two papers published in 1995, we found that small but measurable fast optical signals (i.e., optical signals tracking neural activation frequencies exceeding $1 \mathrm{~Hz}$ ) could be recorded in the motor ${ }^{25}$ and visual ${ }^{26}$ cortex. Both tasks used contralateral control conditions to demonstrate that the phenomena could be observed only in the cortex contralateral to the stimulus or response (i.e., a response was observed in the right hemisphere when the left visual field, or the left hand, was involved in the task, and vice versa). In fact, the visual stimulation study also used a "top versus bottom" visual field stimulation contrast: in different blocks, the stimulus - a vertical black and white grid inverting every $500 \mathrm{~ms}$-was presented either in the top left, top right, bottom left, or bottom right quadrant of the visual field. This led to predict that a neural response should be observed in contralateral, inverted regions of the occipital cortex (i.e., lower for upper visual field stimulation than for lower visual field stimulation). This spatial specificity demonstrated that the observed phenomenon was locally generated and not related to systemic effects. Further, the spatial specificity indicated that movement artifacts were not likely to be the source of the observed effects, because they would not generate effects localized to the appropriate cortical regions. These original studies were replicated in subsequent studies, all including the contralateral-ipsilateral contrasts (Refs. 18, 27, and 28 for visual cortex, and Ref. 29 for motor cortex), and extended to different cortical regions (first reports: auditory cortex in Ref. 30; somatosensory cortex in Ref. 31; frontal and parietal cortex in Refs. 32-34). This and subsequent work from our group indicated that the fast optical signal (or event-related optical signal, EROS) occurs with a very short-latency from stimulation, similar to that of electrically evoked potentials (see Refs. 35 and 36 for reviews). Several other labs have reported results that are consistent with these observations. ${ }^{37-49}$ One lab has reported inability to record fast optical responses in humans, but their methods (including lack of summary statistics conducted across subjects) differed markedly from those used by our group. ${ }^{50}$

In addition to its temporal characteristics, the fast optical signal has spectral characteristics that are consistent with a scattering but not a deoxygenation origin (such as similar effects at either side of the hemoglobin isobestic point). ${ }^{46,51}$ As such, this signal appears to correspond closely to the scattering signal described by the Grinvald group. ${ }^{2-4}$ In fact, work conducted in animals with implanted optical instruments based on back-scattering methods indicated that it is possible to retrieve scattering signals from the cortex ${ }^{52}$ and hippocampus ${ }^{53}$ with similar temporal properties (see also Ref. 54). However, Radhakrishnan et al. ${ }^{55}$ reported an inability to observe a fast optical signal during visual stimulation from the occipital cortex of a monkey. This finding is somewhat puzzling given the rest of the evidence collected in the last 30 years but may perhaps be attributed to the particular methods used in that study (use of a diffuse optical approach but with sources and detectors located very close to the cortical surface, leading to maximum sensitivity for the region underneath the cortex, rather than the cortex itself, which in the visual cortex is particularly thin $-2 \mathrm{~mm}$ or less).

The fast optical signal is typically attributed to a reduction in scattering due to the slight swelling of neurons that occur when they are depolarized. ${ }^{54}$ This has been demonstrated in isolated neurons ${ }^{56}$ and neural fibers, ${ }^{57}$ further demonstration of a change in scattering during neural activity has been reported with optical coherence tomography. ${ }^{58}$ Finally, Lee and Kim ${ }^{59}$ developed 
a biophysical model of how the movement of water associated with membrane depolarization might result in neuronal swelling and consequent scattering changes.

The TR of the fast signal is mostly limited by the sampling rate used in the recording. For instance, when the human cortex is stimulated via transcranial magnetic stimulation (TMS), the fast signal is recorded starting at the very first recording time point (latency: $8 \mathrm{~ms}$ ) after the TMS pulse is applied..$^{60}$ Also, this same study and follow-up work ${ }^{61}$ show that the fast optical signal can be used to investigate the propagation of this initial local activation to other cortical regions, with a latency consistent with the presumed transmission time among cortical regions. Thus, the fast optical signal can be used to track the sequential activation of brain regions. This finding is consistent with the close association between the latency of electrically evoked potentials and of fast optical signals. ${ }^{27}$

The spatial resolution of fast optical signals is related to the methods used for its measurement. Most published work on fast optical imaging (FOI), such as that coming from our lab (reviewed in Ref. 36) and that of Penney's lab, ${ }^{42,43}$ is based on the high-density recording of measures of changes of photon delay during the migration process [obtained with frequencydomain (FD) instrumentation]. Analysis of the relative independence of observations at nearby spatial locations suggests that these measures may reach a spatial resolution of 5 to $10 \mathrm{~mm} .{ }^{18}$ In contrast, measures obtained using light intensity estimates [such as those that can be obtained with continuous-wave (CW) equipment] provide a spatial resolution of only $2 \mathrm{~cm}$, similar to the fNIRS measures (e.g., Refs. 41, 45, and
62). The combination of spatial and TR offered by the fast signal, compared to that offered by other technologies, such as fMRI, electroncephalography (EEG), event-related brain potentials (ERPs) and magnetoencephalography (MEG), makes it an interesting methodology for studying the dynamics of brain activation and of functional connectivity across brain regions, with the added advantage of being compatible for concurrent recording with these methods for comparison and integration purposes. Its main limitations are (a) the limited penetration (as for all diffuse optical imaging methods) and (b) its low SNR - requiring the averaging of a large number of trials $(>50)$ to obtain measurable responses.

Examples of the spatial distributions and time courses of the fNIRS and fast optical (EROS) signals elicited by visual stimulation are presented in Fig. 2 (data from Ref. 63). The paradigm employed in this study involved periods of visual stimulation lasting $\sim 20 \mathrm{~s}$, obtained by reversing a full field, black-andwhite vertical grid pattern at frequencies varying between 1 and $8 \mathrm{~Hz}$, in 19 young adults. The fNIRS analyses show a slow, sustained response encompassing most of the stimulation period, and characterized by an increase in oxy-hemoglobin concentration and a reduction in deoxy-hemoglobin concentration (both consistent with an increase in blood flow), beginning at about $4 \mathrm{~s}$ after the onset of the stimulation period. The response was visible in both striate cortex (BA 17, indicated by the green borders in the figure) and extrastriate cortex (BA 18 and BA 19; lateral to the striate cortex borders). In contrast, the fast optical response (an increase in photon delay indicating a greater penetration of light into tissue consistent with

Hemodynamic response to repeated visual stimulations measured with fNIRS

(a)

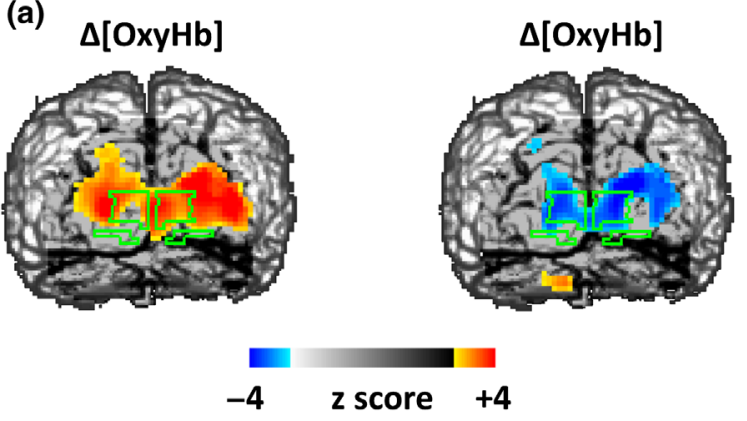

(b)

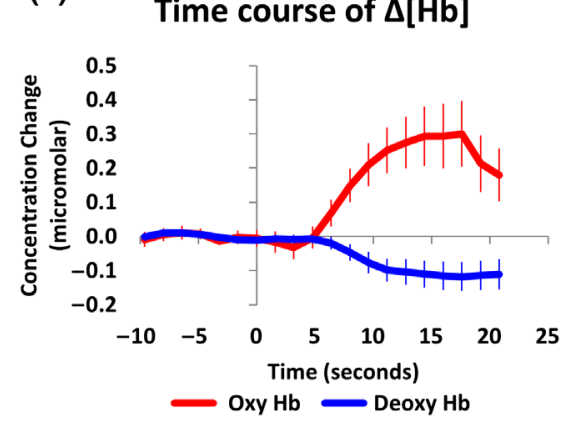

Neuronal response to individual visual stimulation measured with EROS
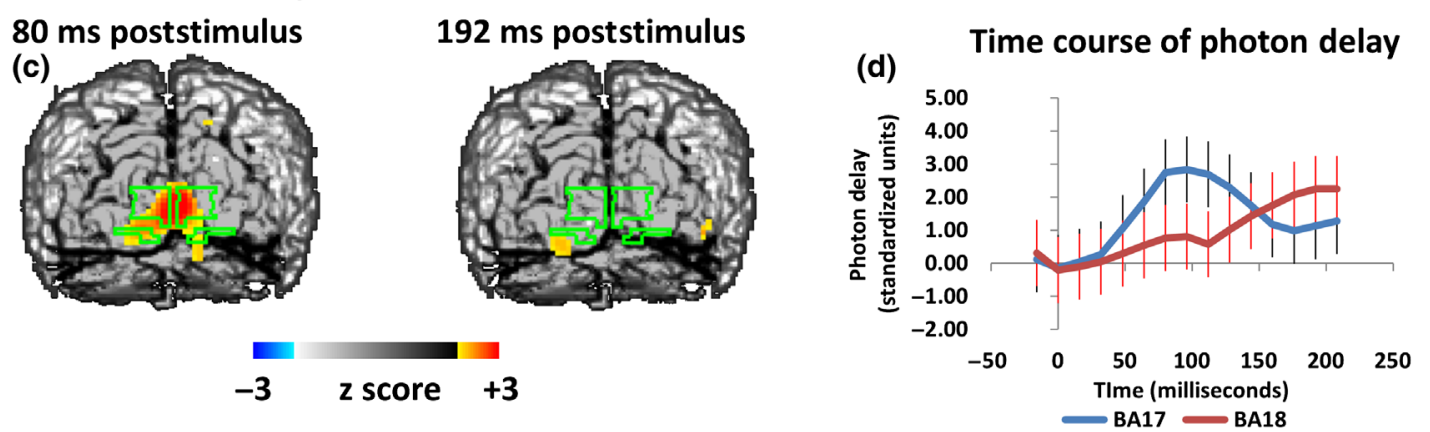

Fig. 2 (a) Maps and (b) time course of the fNIRS hemodynamic response (measured as a change in the concentration of oxy- and deoxy-hemoglobin in occipital regions) during repeated visual stimulation in 19 young adults. (c) Maps and (d) time course of the fast optical signal (EROS-measured as a change in the delay of near-infrared photons migrating through occipital regions) during repeated visual stimulation measured concurrently with the fNIRS response in the same subjects. For the EROS activity, the two maps refer to two different latencies $(80$ and $192 \mathrm{~ms}$ ) from the individual stimulus onset (grid reversals). 
a reduction in light scattering) had a very fast onset $(80 \mathrm{~ms})$ in striate cortex and a relatively slower onset (192 ms) in extrastriate cortex. Note that the EROS activity identifies two separate responses (occurring at different locations and different latencies) to the visual stimulation. These two responses could not be separated with the fNIRS measurements. Table 1 reports a list of milestone papers in human FOI research. Table 2 presents a comparison of animal and human techniques for measuring intrinsic functional signals from the brain.

The fast optical signal has great potential as a research tool for measuring in parallel the time course of neural activity in different cortical regions. This may provide information about the order of activation of these regions. It can also provide important information about functional connectivity, and, when paired with localized stimulation methods, such as TMS, it may also provide useful information about effective connectivity (i. e., causal links between the activities in different regions). This may potentially be of significant utility for developing full brain models of psychological function. Because of its very minimal invasivity, it can be used in many populations, including preterm and at-term infants, making it possible to study functional signals across a wide variety of age ranges.

The vast majority of fast signal studies have used photon delay as the method for revealing changes in infrared light scattering that occur with neural activity. Photon delay measures have some advantages over intensity measures (greater penetration and higher spatial resolution), which can be particularly important for obtaining measures of neural activity from the brain with a combination of high spatial and TR. However, some labs (e.g., Refs. 36-40 and 44) have shown that intensity

Table 1 Milestone papers on FOS.

\begin{abstract}
First demonstration of FOS in visual cortex

First demonstration of FOS in motor cortex

First demonstration of FOS in auditory cortex

First demonstration of FOS in somatosensory cortex
\end{abstract}

First demonstration of FOS in secondary cortices

First demonstration of oscillatory FOS

First full head recording of FOS

First FOS long-distance lagged cross-correlations

First concurrent recording of FOS and EEG/ERPs

First concurrent recording of FOS and fMRI

First concurrent use of FOS and TMS
Table 2 Comparison of human and animal intrinsic functional optical imaging.

\begin{tabular}{lccc}
$\begin{array}{l}\text { Feature } \\
\text { Penetration }\end{array}$ & $\begin{array}{c}\text { Animal: } \\
\text { hemodynamic }\end{array}$ & $\begin{array}{c}\text { Human: } \\
\text { fast signal }\end{array}$ & $\begin{array}{c}\text { Human: } \\
\text { fNIRS }\end{array}$ \\
$\begin{array}{l}\text { Best spatial } \\
\text { resolution }\end{array}$ & 50 to $100 \mu \mathrm{mm}$ & 20 to $30 \mathrm{~mm} 20$ to $30 \mathrm{~mm}$ \\
$\begin{array}{l}\text { Best TR } \\
\text { SNR }\end{array}$ & $1 \mathrm{~ms}$ & $8 \mathrm{~ms}$ & $5 \mathrm{~s}$ \\
$\begin{array}{l}\text { Invasitivity } \\
\text { High }\end{array}$ & Low & Medium \\
$\begin{array}{l}\text { Portability } \\
\begin{array}{l}\text { Compatibility with } \\
\text { concurrent electrical } \\
\text { and MR recordings }\end{array}\end{array}$ & Good to Low & Gow $20 \mathrm{~mm}$ & Low \\
\hline
\end{tabular}

measures may also be used to record fast signals. The main advantage of intensity measures is that they can be obtained with CW instruments, which are cheaper and more widely available than the FD instruments required for photon delay measures. However, intensity measures are likely to provide reduced spatial resolution relative to photon delay measures. In any case, to obtain high spatial resolution, it is very helpful to use high-density recordings (i.e., recordings with more than one channel every 4 to $5 \mathrm{~cm}^{2}$ ). This requires large instruments (capable of recording from several hundred channels), unless only small areas of the brain are investigated ("patch" recordings).

\section{Optical Pulse Signal: from Blood Vessels to Brain}

The pulsation of arteries in the head generates an oscillatory signal whose carrier frequency is determined by the heart rate (see Fig. 3). In most functional optical brain imaging studies, this signal (which is quite large-up to orders of magnitude larger than the oxygenation and fast signals) is considered a nuisance variable and eliminated with appropriate procedures (e.g., Ref. 65). However, we ${ }^{64}$ have recently shown that the optical pulsation signal, or pulse-DOT (if measured using a sampling rate exceeding $20 \mathrm{~Hz}$ ), in fact provides very useful data about the state of cerebral arteries. First, consistent with its arterial origin, the signal is oxygen-saturated $>95 \%$. Second, the amplitude of pulse oscillations (which is related to the actual volume change during a pulsation) is larger when the measures are taken over large arteries running along the surface of the cortex (such as tracts of the anterior, middle, and posterior cerebral arteries). The amplitude of the oscillations is related (across subjects) to pulse pressure (i.e., the difference between systolic and diastolic systemic blood pressure measured at the forearm) and can therefore be considered as an index of pulse pressure in the brain. Third, the timing of the systolic peak (and in particular, the difference in timing between the systolic peaks at different points along a cerebral artery) can be used to measure pulse wave velocity (i.e., the speed of propagation of the arterial pulse along an artery) inside the brain. Fourth, measures of the shape of the pulse (which we have labeled "arterial compliance" or "pulse relaxation function") can be used to study the 
(a)

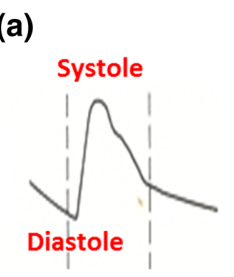

(b)

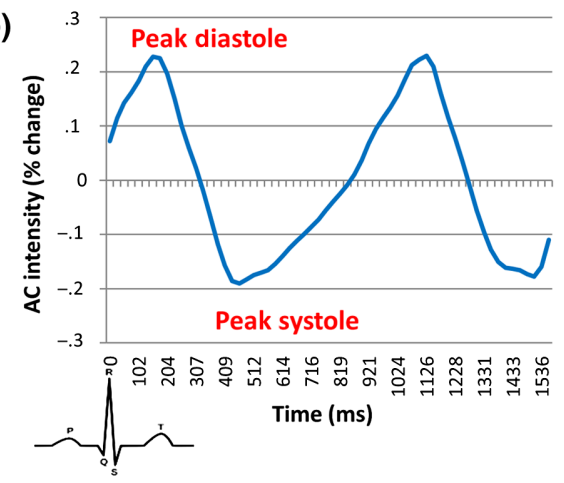

(e)

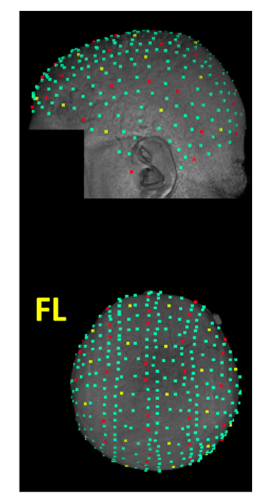

(c)
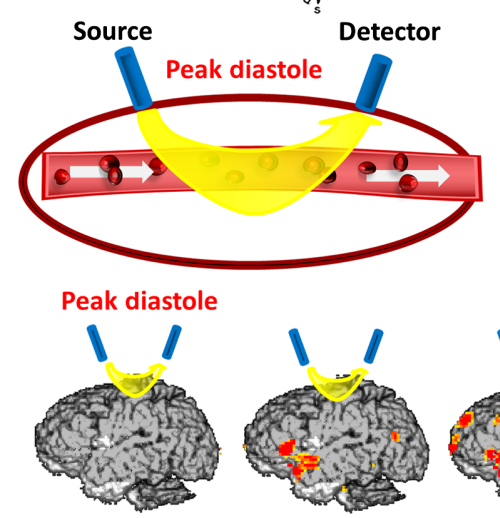

$153 \mathrm{~ms}$

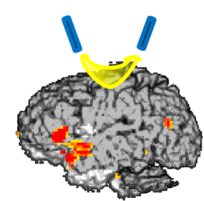

$204 \mathrm{~ms}$

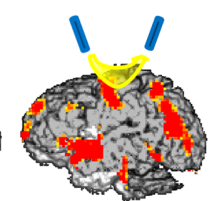

$255 \mathrm{~ms}$

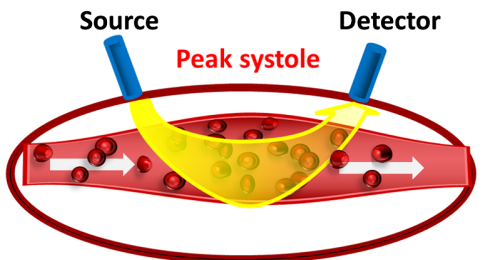

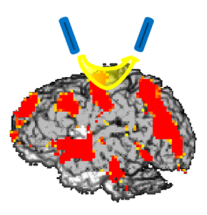

$307 \mathrm{~ms}$
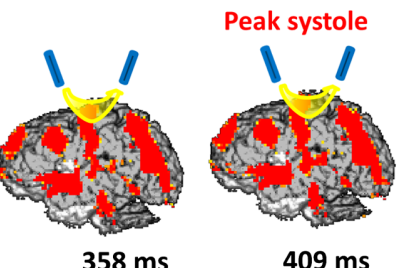

$409 \mathrm{~ms}$

Fig. 3 (a) Typical pulse wave as measured at the carotid by ultrasound Doppler. (b) Pulse wave measured optically as a change in the intensity of the AC light moving between a source and a detector located on the surface of the head. The peak of the systole corresponds to a minimum (and the peak of the diastole to a maximum) in the amount of light detected after traveling through the tissue. (c) Schematic depiction of changes in arterial diameter and oxy-hemoglobin content during a pulse cycle, causing the changes in light intensity shown in (b), as more light is absorbed during the systole. (d) Spatially filtered images of the pulse in the brain overtime averaged across participants, emphasizing the largest arteries. Images are based on changes from the diastolic peak value (on average $153 \mathrm{~ms}$ after onset of the EKG R-wave). The systolic peak (on average $409 \mathrm{~ms}$ after R-wave onset) shows the maximum change with respect to the diastolic peak value. At this point, the large arteries are most visible. (e) Distribution of the sources (in yellow) and detectors (in red) used for data collection over the MRrendered scalp of a representative participants. Other digitized locations used for coregistration with the structural MRI recordings are shown in green. $\mathrm{FL}=$ front left. Figure reprinted from Ref. 64 with permission from the publisher (Wiley).

way in which arteries relax after a systole. Using the Windkessel model (named from a word that means "air chamber" in German and describing the shape of the arterial pulse pressure waveform as a result of the interaction of stroke volume and compliance of the large, elastic arteries) and other biophysical models of arterial function, these measures can be used to study the elasticity of the arterial walls (or lack thereof; i.e., arterial stiffness or arteriosclerosis). Thus, mapping parameters of the arterial pulse from different brain locations can be used for mapping stiffness of the cerebral arteries, with higher pulse amplitude, lower pulse relaxation function, and faster transit times indicating less elastic cerebral arteries.

We applied this approach to study the correlates of arterial health in a large sample of normally aging healthy adults (age range $=55$ to 87 ), none of whom had any history of stroke or other major health problems. ${ }^{64}$ In this sample, as predicted, pulse amplitude was positively correlated with age; the pulse relaxation function was instead positively correlated with measures of cardiorespiratory fitness (CRF) (see Fig. 4), but also with measures of cortical volume, white matter volume, and total cortical volume, indicating that more elastic arteries are associated with more preserved brain tissue in aging. Pulse wave velocity (or transit time) in the left middle cerebral artery, feeding Broca's area, was correlated with preservation of verbal fluency, whereas pulse wave velocity in the precentral artery feeding dorsolateral prefrontal cortex bilaterally was correlated with operational working memory span (with a double-dissociation between these two cognitive measures).

In a subsequent study performed in a separate independent sample ${ }^{66}$ we replicated these findings and extended them to a wider age range (18 to 75 years). Further analyses of these data ${ }^{67}$ also showed that 3-D reconstruction of the pulse data yielded improvements in the reliability of the pulse parameter $(r>0.98$ for both pulse amplitude and pulse relaxation function), and demonstrated that the relationship between pulse relaxation function and cortical volume emerges after age 48 , and is specific to each particular brain region. In other words, for each brain region, the extent of brain tissue atrophy is related to the stiffness of the arteries in that region (both across and within subjects). In addition, arterial stiffness is correlated with white matter damage, as indicated by MRI measures (structural and diffusion tensor imaging). This is consistent with the idea 


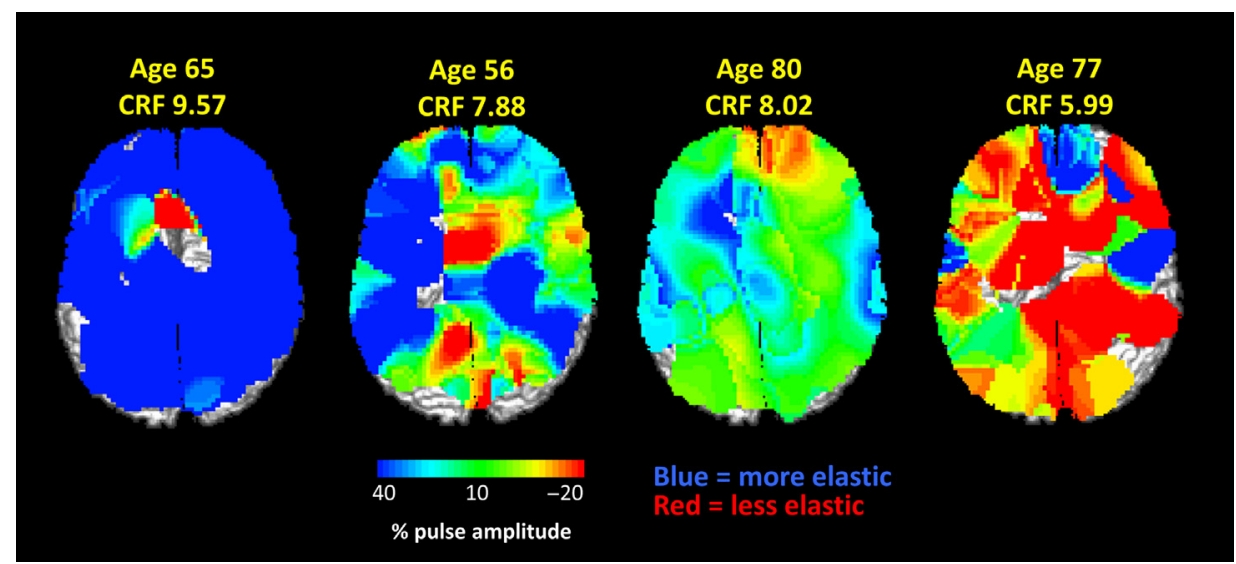

Fig. 4 Maps of the arterial pulse relaxation function obtained with diffuse optical imaging methods in four healthy adults varying in age and CRF. Figure reprinted from Ref. 64 with permission from the publisher (Wiley).

that measures of arterial stiffness obtained with optical methods are sensitive to small brain vessels disease, a possible precursor of vascular dementia. ${ }^{68}$

In a separate study based on our original 55- to 87-year-old sample ${ }^{69}$ we also investigated whether pulse-DOT parameters were sensitive to cerebral vasodilation and vasoconstriction manipulations. Vasodilation should generate a reduction in peripheral resistance, and as such it should also be associated with a reduction of pulse amplitude in the brain. This drop may be more evident for the arteries feeding the cortical region in which the vasodilation occurs. We used two forms of manipulation designed to generate cerebral vasodilation: breath-holding, to generate hypercapnia and global cerebral vasodilation, and a cognitive visual working memory task, designed to generate vasodilation in the visual cortex and fronto-parietal regions associated with working memory function. The results of the study showed that both manipulations produced changes in pulse amplitude. These changes appeared to be related to regions of the brain in which vasodilation occurred, being more generalized in the breath-holding task, and more specific for task-related cortical regions during the working memory task.

\section{Neurovascular Coupling: from Brain to Blood Vessels}

An important issue in brain imaging is the link that exists between neural activity and vascular function (i.e., neurovascular coupling, investigated extensively by the Grinvald group, see, for example, Ref. 70), and in particular the shape of the relationship between the amount of neural activity in a particular cortical region and the amount of increase in oxygenation (or BOLD response) occurring in the same area. This relationship is important because, in many cases, we use measures related to the oxygenation response (such as the amplitude of the BOLD fMRI response or of the fNIRS activity) to infer the existence of neural activity, not only in qualitative but often in quantitative terms. This is particularly critical when using linear decomposition techniques, such as the general linear model, to decompose the hemodynamic signal into different subcomponents. In this case, linearity of the relationship between neural activity and vascular response needs to be assumed.

Several studies have analyzed the shape of this relationship and the majority of them are based on conditions in which the stimulus is parametrically varied. For instance Fox and Raichle ${ }^{71}$ measured the amplitude of the hemodynamic response (in that case measured using $\mathrm{O}^{15}$-PET) under conditions in which visual stimulation frequency was parametrically varied. They reported a linear relationship between stimulus frequency and the amplitude of the hemodynamic response-an observation that had a critical role in the development of subtraction methods for the analysis of neuroimaging data. It should be noted, however, that in this study there was no direct measure of neural activity. It was assumed that this activity should increase linearly with the frequency of stimulation-but there was no verification of this assumption. During the last 30 years, other studies have investigated the assumption of linearity of neurovascular coupling (e.g., Refs. 72 and 73), and several of them have added measures of neural function to the hemodynamic measures (such as the Ref. 72). In the vast majority of these papers, the measures of neural function are obtained using electrophysiological recordings (EEG/ERPs, e.g., Refs. 72 and 74). Although informative, these studies have the problem that the measures of neural and vascular function may be spatially nonoverlapping - that is, they may not refer to the same cortical region.

Diffuse optical imaging methods provide a very interesting opportunity for studying neurovascular coupling: they can simultaneously provide measures of neural activity (through the fast signal) and measures of hemodynamic function (the oxygenation response using fNIRS methods). Not only can these measures be obtained at the same time, but they can also be obtained from the same space (i.e., through the same sets of sources and detectors, measuring photons moving through the same cortical regions). fNIRS measures are somewhat quantitative in nature, as they provide estimates of the changes in concentration of oxy- or deoxy-hemoglobin in a particular region. Unfortunately, the most-commonly used procedures for measuring the fast signal (measures of phase delay) are not similarly quantitative, nor it is clear what a quantitative measure of neural activity should exactly be. For this reason, it may be useful to also obtain estimates of neural activity obtained with other methods, such as ERPs, to determine the generalizability of the conclusions.

Therefore in two separate experiments (a smaller one, based on eight young adults ${ }^{75}$ and a larger one based on a large sample comprising younger and older adults varying in CRF), ${ }^{63}$ we 
estimated the shape of the neurovascular coupling function comparing the amplitudes of the neural and vascular responses to visual stimulations of varying frequency in primary visual cortex. In both cases, the results obtained with the fast optical response and with the ERP measures were very similar. Both showed a response that attenuated approximately linearly with the logarithm of the visual stimulation frequency (also the latencies of the initial responses were consistent across the two techniques, peaking at $\sim 80$ to $100 \mathrm{~ms}$ - although the ERP responses showed several subsequent peaks at longer latencies, presumably indicating the recruitment of extrastriate cortical areas).

In both studies, the hemodynamic response measured with fNIRS methods appeared to increase slightly from the lowest to the highest frequencies of stimulation; this is consistent with previous work, such as the Fox and Raichle's paper. ${ }^{71}$ In the second larger study, the experiment was repeated twice with the same participants, with ERPs, the fast optical signal and fNIRS recorded concurrently, and with BOLD fMRI obtained in a separate session, and showing effects similar to the fNIRS measures.

Although these studies showed different effects as a function of increases in stimulation frequency for the neuronal measures (a reduction) and hemodynamic measures (an increase), this per se does not invalidate the idea that hemodynamic phenomena are directly related to neural activity. In fact, given the relatively high stimulation frequency used in the study (from 1 up to $8 \mathrm{~Hz}$ ), the individual hemodynamic responses generated by each stimulus presentation should be expected, because of their very slow time course, to summate. We should therefore expect that, if linearity holds, the hemodynamic response should be proportional to the integration overtime of the neural response.

In fact, in both studies the relationship between the integrated neuronal activity and hemodynamic (fNIRS) responses appeared to be somewhat nonlinear, with some evidence of saturation for higher stimulation frequency conditions. In the first study, based on a small sample size, this departure from linearity, however, was not significant, probably because of very large confidence intervals. In the second study, however, with a much larger sample size, the relationship was clearly nonlinear. A much better fit was obtained when a root square relationship between the neural and the hemodynamic responses was used.

The larger age and CRF ranges used in the second study also allowed us to determine whether these variables impact the neurovascular coupling function. In fact, the data suggested that younger and highly fit older adults have relatively similar neurovascular coupling functions. However, low-fit older adults had a markedly reduced slope of the neurovascular coupling function (but a similarly shaped function to other two groups). These data suggest that low levels of CRF may reduce the ability of older adults to vasodilate as a function of the same amount of neural activity. This result is consistent with the observation that poor CRF may be associated with reduced vascular reactivity (e.g., Ref. 76).

\section{Conclusions}

The work of Grinvald, and in particular the development of intrinsic optical methods for studying neural and cerebrovascular function, has greatly influenced the field of brain imaging. Here, we have reviewed examples of this influence in the area of diffuse optical imaging and in our own work in humans. This work spans areas such as functional brain imaging, cognitive neuroscience, functional connectivity, influence of arterial health on cortical structure and function, and neurovascular coupling. All these areas profit from the recognition that intrinsic changes in optical properties can be used to retrieve signals related to neuronal and hemodynamic function, all areas for which Grinvald has made crucial and long-lasting contributions.

\section{Disclosures}

None of the authors has financial interests in any of the companies manufacturing the equipment used to collect data mentioned in this paper.

\section{Acknowledgments}

This paper was supported by National Institute of Mental Health (NIMH) Grant No. 5R56MH097973 (G. Gratton and M. Fabiani, PIs), National Institute on Aging (NIA) Grant No. 1RC1AG035927 (M. Fabiani, PI), and National Center for Research Resources (NCRR) Grant No. S10-RR029294 (G. Gratton PI). All the human data mentioned in this study were collected using procedures approved by the Institutional Review Board of the University of Illinois and in compliance with the Helsinki Convention and National Institute of Health (NIH) directives.

\section{References}

1. A. Grinvald et al., "Functional architecture of cortex revealed by optical imaging of intrinsic signals," Nature 324, 361-364 (1986).

2. R. D. Frostig et al., "Cortical functional architecture and local coupling between neuronal activity and the microcirculation revealed by in vivo high-resolution optical imaging of intrinsic signals," Proc. Natl. Acad. Sci. U. S. A. 87(16), 6082-6086 (1990).

3. D. Malonek and A. Grinvald, "Interactions between electrical activity ad cortical microcirculation revealed by imaging spectroscopy: implication for functional brain mapping," Science 272, 551-554 (1996).

4. D. Malonek et al., "Vascular imprints of neuronal activity: relationships between the dynamics of cortical blood flow, oxygenation, and volume changes following sensory stimulation," Proc. Natl. Acad. Sci. U. S. A. 94(26), 14826-14831 (1997).

5. A. Grinvald et al., "Imaging the neocortex functional architecture using multiple intrinsic signals: implications for hemodynamic-based functional imaging," in Imaging in Neuroscience: A Laboratory Manual, F. Helmchen and A. Konnerth, Eds., CSHL Press, Cold Spring Harbor, New York (2011).

6. C. H. Chin-Bee et al., "The triphasic intrinsic signal: implications for functional imaging," J. Neurosci. 27(17), 4572-4586 (2007).

7. A. J. Kennerley et al., "Is optical imaging spectroscopy a viable measurement technique for the investigation of the negative BOLD phenomenon? A concurrent optical imaging spectroscopy and fMRI study at high field (7 T)," NeuroImage 61, 10-20 (2012).

8. C. Martin et al., "Investigating neural-hemodynamic coupling and the hemodynamic response function in the awake rat," NeuroImage 32, 33-48 (2006).

9. D. Berwick et al., "Fine detail of neurovascular coupling revealed by spatiotemporal analysis of the hemodynamic response to single whisker stimulation in rat barrel cortex," J. Neurophysiol. 99, 787-798 (2008).

10. Y. B. Sirotin et al., "Spatiotemporal precision and hemodynamic mechanism of optical point spreads in alert primates," Proc. Natl. Acad. Sci. U. S. A. 106(43), 18390-18395 (2009).

11. A. Devor et al., "Frontiers in optical imaging of cerebral blood flow and metabolism," J. Cereb. Blood Flow Metab. 32(7), 1259-1276 (2012).

12. F. F. Jobsis, "Noninvasive, infrared monitoring of cerebral and myocardial oxygen sufficiency and circulatory parameters," Science 198(4323), 1264-1267 (1977).

13. D. A. Boas, A. M. Dale, and M. A. Franceschini, "Diffuse optical imaging of brain activation: approaches to optimizing image sensitivity, resolution, and accuracy," Neurolmage 23, S275-S288 (2004). 
14. A. M. Chiarelli et al., "Combining energy and Laplacian regularization to accurately retrieve the depth of brain activity of diffuse optical tomographic data," J. Biomed. Opt. 21(3), 036008 (2016).

15. M. Ferrari, L. Mottola, and V. Quaresima, "Principles, techniques, and limitations of near infrared spectroscopy," Can. J. Appl. Physiol. 29(4), 463-487 (2004)

16. A. T. Eggebrecht et al., "Mapping distributed brain function and networks with diffuse optical tomography," Nat. Photonics 8(6), 448-454 (2014).

17. T. Nishimura et al., "Descent of the larynx in chimpanzee infants," Proc. Natl. Acad. Sci. U. S. A. 100, 6930-6933 (2003).

18. G. Gratton and M. Fabiani, "The event-related optical signal (EROS) in visual cortex: replicability, consistency, localization, and resolution," Psychophysiology 40(4), 561-571 (2003).

19. Y. Hoshi and M. Tamura, "Detection of dynamic changes in cerebral oxygenation coupled to neuronal function during mental work in man," Neurosci. Lett. 150(1), 5-8 (1993)

20. S. Ogawa et al., "Functional brain mapping by blood oxygenation level-dependent contrast magnetic resonance imaging. A comparison of signal characteristics with a biophysical model," Biophys. J. 64(3), 803-812 (1993).

21. M. Ferrari and V. Quaresima, "A brief review on the history of human functional near-infrared spectroscopy (fNIRS) development and fields of application," NeuroImage 63(2), 921-935 (2012).

22. X. Zhang et al., "The study of cerebral hemodynamic and neuronal response to visual stimulation using simultaneous NIR optical tomography and BOLD fMRI in humans," Proc. SPIE 5686, 566 (2005).

23. J. V. Lee et al., "Comparison of responses in awake and anesthetized zebra finches to birdsong measured by noninvasive diffuse optical imaging," J. Comp. Physiol. A 199(3), 227-238 (2013).

24. E. Yacoub et al., "Investigation of the initial dip in fMRI at 7 Tesla," NMR Biomed. 14(7-8), 408-412 (2001).

25. G. Gratton et al., "Rapid changes of optical parameters in the human brain during a tapping task," J. Cognit. Neurosci. 7(4), 446-456 (1995).

26. G. Gratton et al., "Shades of gray matter: noninvasive optical images of human brain reponses during visual stimulation," Psychophysiology 32(5), 505-509 (1995).

27. G. Gratton et al., "Fast and localized event-related optical signals (EROS) in the human occipital cortex: comparisons with the visual evoked potential and fMRI," NeuroImage 6(3), 168-180 (1997).

28. G. Gratton et al., "Memory-driven processing in human medial occipital cortex: an event-related optical signal (EROS) study," Psychophysiology 35(3), 348-351 (1998).

29. M. C. DeSoto et al., "When in doubt, do it both ways: brain evidence of the simultaneous activation of conflicting responses in a spatial Stroop task," J. Cognit. Neurosci. 13(4), 523-536 (2001).

30. T. Rinne et al., "Scalp-recorded optical signals make sound processing from the auditory cortex visible," NeuroImage 10, 620-624 (1999).

31. E. L. Maclin et al., "The event-related optical signal to electrical stimulation of the median nerve," NeuroImage 21(4), 1798-1804 (2004).

32. K. A. Low et al., "Fast optical imaging of frontal cortex during active and passive oddball tasks," Psychophysiology 43, 127-136 (2006).

33. C. Y. Tse et al., "Imaging the cortical dynamics of language processing with the event-related optical signal (EROS)," Proc. Natl. Acad. Sci. U. S. A. 104, 17157-17162 (2007).

34. C. Y. Tse et al., "Rules rule! Brain activity dissociates the representations of stimulus contingencies with varying levels of complexity," J. Cognit. Neurosci. 24(9), 1941-1959 (2012).

35. G. Gratton and M. Fabiani, "The event-related optical signal: a new tool for studying brain function," Int. J. Psychophysiol. 42(2), 109-121 (2001).

36. G. Gratton and M. Fabiani, "Fast optical imaging of human brain function," Front. Hum. Neurosci. 4(9), 52 (2010).

37. M. Wolf et al., "Functional frequency-domain near-infrared spectroscopy detects fast neuronal signal in the motor cortex," NeuroImage 17(4), 1868-1875 (2002).

38. M. Wolf et al., "Fast cerebral functional signal in the 100-ms range detected in the visual cortex by frequency-domain near-infrared spectrophotometry," Psychophysiology 40(4), 521-528 (2003).

39. M. Wolf et al., "Detection of the fast neuronal signal on the motor cortex using functional frequency domain near infrared spectroscopy," $A d v$. Exp. Med. Biol. 510, 193-197 (2003).
40. G. Morren et al., "Detection of fast neuronal signals in the motor cortex from functional near infrared spectroscopy measurements using independent component analysis," Med. Biol. Eng. Comput. 42(1), 92-99 (2004).

41. M. A. Franceschini and D. A. Boas, "Noninvasive measurement of neuronal activity with near-infrared optical imaging," NeuroImage 21(1), 372-386 (2004).

42. C. Y. Tse and T. B. Penney, "Preattentive change detection using the event-related optical signal," IEEE Eng. Med. Biol. Mag. 26(4), 52-58 (2006).

43. C. Y. Tse and T. B. Penney, "On the functional role of temporal and frontal cortex activation in passive detection of auditory deviance," NeuroImage 41(4), 1462-1470 (2008).

44. M. Kubota et al., "Fast (100-175 ms) components elicited bilaterally by language production as measured by three-wavelength optical imaging," Brain Res. 1226, 124-133 (2008).

45. A. V. Medvedev et al., "'Seeing' electroencephalogram through the skull: imaging prefrontal cortex with fast optical signal," J. Biomed. Opt. 15(6), 061702 (2010).

46. J. Lee and S. J. Kim, "Spectrum measurement of fast optical signal of neural activity in brain tissue and its theoretical origin," NeuroImage 51(2), 713-722 (2010).

47. A. M. Chiarelli et al., "Fast optical signal in visual cortex: improving detection by general linear convolution model," NeuroImage $\mathbf{6 6}$, 194-202 (2013).

48. A. M. Chiarelli et al., "Fast optical signals in the sensorimotor cortex: general linear convolution model applied to multiple source-detector distance-based data," NeuroImage 85, 245-254 (2014).

49. H. D. Lu et al., "Intrinsic signal optical imaging of visual brain activity: tracking of fast cortical dynamics," NeuroImage 148, 160-168 (2017).

50. J. Steinbrink et al., "The fast optical signal-robust or elusive when non-invasively measured in the human adult?" NeuroImage 26(4), 996-1008 (2005).

51. G. Gratton et al., "Effects of measurement method, wavelength, and source-detector distance on the fast optical signal," NeuroImage 32, 1576-1590 (2006)

52. D. M. Rector et al., "Spatio-temporal mapping of rat whisker barrels with fast scattered light signals," NeuroImage 26(2), 619-627 (2005).

53. D. M. Rector et al., "Imaging the dorsal hippocampus: light reflectance relationships to electroencephalographic patterns during sleep," Brain Res. 696(1-2), 151-160 (1995).

54. A. J. Foust and D. M. Rector, "Optically teasing apart neural swelling and depolarization," Neuroscience 145(3), 887-899 (2007).

55. H. Radhakrishnan et al., "Fast optical signal not detected in awake behaving monkeys," NeuroImage 45(2), 410-419 (2009).

56. L. B. Cohen, R. D. Keynes, and D. Landowne, "Changes in light scattering that accompany the action potential in squid giant axons: potential-dependent components," J. Physiol. 224(3), 701-725 (1972).

57. R. A. Stepnoski et al., "Noninvasive detection of changes in membrane potential in cultured neurons by light scattering," Proc. Natl. Acad. Sci. U. S. A. 88(21), 9382-9386 (1991).

58. J. Lee and D. A. Boas, "Frequency-domain measurement of neuronal activity using dynamic optical coherence tomography," in Anпиаl Int. Conf. of the IEEE Engineering in Medicine and Biology Society (EMBC'12), pp. 2643-2646 (2012).

59. J. Lee and S. J. Kim, "Spectrum measurement of fast optical signal of neural activity in brain tissue and its theoretical origin," Neurolmage 51(2), 713-722 (2010).

60. N. A. Parks et al., "Examining cortical dynamics and connectivity with concurrent simultaneous single-pulse transcranial magnetic stimulation and fast optical imaging," NeuroImage 59(3), 2504-2510 (2012).

61. N. A. Parks et al., "The influence of posterior parietal cortex on extrastriate visual activity: a concurrent tms and fast optical imaging study," Neuropsychologia 78, 153-158 (2015).

62. A. V. Medvedev et al., "Event-related fast optical signal in a rapid object recognition task: improving detection by the independent component analysis," Brain Res. 1236, 145-158 (2008).

63. M. Fabiani et al., "Neurovascular coupling in normal aging: a combined optical, ERP and fMRI study," NeuroImage 85(Pt. 1), 592-607 (2014).

64. M. Fabiani et al., "Taking the pulse of aging: mapping pulse pressure and elasticity in cerebral arteries with diffuse optical methods," Psychophysiology 51, 1072-1088 (2014). 
65. G. Gratton and P. M. Corballis, "Removing the heart from the brain: compensation for the pulse artifact in the photon migration signal," Psychophysiology 32, 292-299 (1995).

66. C. H. Tan et al., "Mapping cerebral pulse pressure and arterial compliance over the adult lifespan with optical imaging," PLoS One 12(2), e0171305 (2017)

67. A. M. Chiarelli et al., "Regional optical measures of cerebrovascular status associated with cortical volume in healthy aging," in Paris fNIRS Conf. (2016).

68. J. Ewoud et al., "Progression of cerebral small vessel disease in relation to risk factors and cognitive consequences: Rotterdam scan study," Stroke 39, 2712-2719 (2008).

69. C. H. Tan et al., "Optical measures of changes in cerebral vascular tone during voluntary breath holding and a Sternberg memory task," Biol. Psychol. 118, 184-194 (2016).

70. I. Vanzetta and A. Grinvald, "Coupling between neuronal activity and microcirculation: implications for functional brain imaging," HFSP J. 2, 79-98 (2008).

71. P. T. Fox and M. E. Raichle, "Stimulus rate determines regional brain blood flow in striate cortex," Ann. Neurol. 17(3), 303-305 (1985).

72. H. Obrig et al., "Habituation of the visually evoked potential and its vascular response: implications for neurovascular coupling in the healthy adult," NeuroImage 17(1), 1-18 (2002).

73. N. Zhang et al., "Linearity of blood-oxygenation-level dependent signal at microvasculature," NeuroImage 48(2), 313-318 (2009).

74. M. Sood et al., "NIRS-EEG joint imaging during transcranial direct current stimulation: online parameter estimation with an autoregressive model," J. Neurosci. Methods 274, 71-80 (2016).

75. G. Gratton, M. R. Goodman-Wood, and M. Fabiani, "Comparison of neuronal and hemodynamic measure of the brain response to visual stimulation: an optical imaging study," Hum. Brain Mapp. 13, 13-25 (2001).
76. T. Tarumi et al., "Cerebral/peripheral vascular reactivity and neurocognition in middle-age athletes," Med. Sci. Sports Exercise 47(12), 2595-2603 (2015).

Gabriele Gratton received his MD degree from the University of Rome in 1980 and his PhD from the University of Illinois in 1991. $\mathrm{He}$ is a professor of psychology and neuroscience at the University of Illinois. He received the Provost Outstanding Junior Faculty Research Award from the University of Missouri and the Early Career Award from the Society for Psychophysiological Research. He is a fellow of the Association for Psychological Science, and was the president of SPR in 2010.

Antonio M. Chiarelli received his BS and MS degrees in physics engineering, optics and photonics from the Polytechnic in Milan, Italy, in 2006 and 2009, respectively. In 2013, he received his PhD in neuroimaging technologies from University of G. D'Annunzio in Chieti-Pescara, Italy. $\mathrm{He}$ is a research associate in the Cognitive Neuroimaging Lab at the Beckman Institute, University of Illinois. His research interests focus on the functional optical brain imaging methods (including hardware, analytic developments, and applications).

Monica Fabiani received her $\mathrm{PhD}$ in biological psychology from the University of Illinois in 1990. She is a professor of psychology and neuroscience at the University of Illinois, and a faculty at the Beckman Institute. She cochaired the Biological Intelligence Group at the Beckman Institute (2005 to 2010) and was the president of the Society for Psychophysiological Research (2007 to 2008). She is the editor of the Journal Psychophysiology, and a fellow of the Association for Psychological Science. 